It really does seem hard to believe that successful unorthodox treatments are suppressed because they are not patentable. If they were good then they would have been adopted in countries other than the USA where the allegations of corruption levelled by Moss do not apply. Moreover, Moss mentions, but gives scant attention to the need to protect the public from unscrupulous frauds. There are too many documented cases to ignore the fact that National Institutes of Health, the American Cancer Society and similar bodies have a duty not only to promote research, but also to protect the public from quacks. So the first charge is not entirely proven; nonetheless, Moss's book leaves an uncomfortable feeling that all is not well.

When he turns to cancer prevention (or the lack of it) he is on firmer ground. There is plenty of evidence that in the past, and also at present, some parts of industry have callously ignored the welfare of their workers. Moss revels in the horrors of asbestos and mesothelioma. These facts have been well documented elsewhere, however, and are no longer the main issue. $\mathrm{He}$ is quite wrong in saying that research into preventive methods has been ignored; this only reveals his limited horizons. There is at present a great deal of activity in basic research laboratories and in industrial laboratories to find ways of preventing the widespread use of hazardous chemicals. Again, this needs collaboration between industry and basic research, but the relationship is not always an easy one. Any scientist who helps a company to sort out a problem is likely to be branded a consultant - and therefore suspect - by people such as Moss. This should not be so - the expertise in the universities and research institutes should be available to industry. On the other hand, it does cause concern that one distinguished scientist and government adviser should also be found to be a consultant for six large corporations and two manufacturers' associations.

The book is well written and easy to read. In an era in which monoclonal antibodies and the production of specific polypeptides by genetic manipulation are likely to become big business, it raises issues that have to be faced. The manner in which the issues are raised, however, leaves a rather nasty taste in the mouth.

D. G. Harnden is Professor of Cancer Studies at the University of Birmingham, UK.

\title{
State of the art in nutrition
}

\section{J.W.T. Dickerson}

\begin{abstract}
Human Nutrition-A Comprehensive Treatise. General editors R. B. Alfin-Slater and D. Kritchevsky. (Plenum: 1979/1980). Vol. 1. Nutrition: Pre- and Postnatal Development (edited by M. Winick), pp.495; £24.89, \$39.50. Vol. 2. Nutrition and Growth (edited by D.B. Jelliffe and E.F.P. Jelliffe), pp.450; £23.63, \$37.50. Vol. 3A. Nutrition and the Adult: Macronutrients, pp.290; $£ 15.75, \$ 25$. Vol. 3B. Nutrition and the Adult: Micronutrients, pp.424; $£ 24.89, \$ 39.50$. (Both edited by R.B. Alfin-Slater and D. Kritchevsky.) Vol. 4. Nutrition: Metabolic and Clinical Applications (edited by R.E. Hodges), pp.478; £23.63, \$37.50.
\end{abstract}

THIs large work has grown out of an idea "to provide the research investigator and the advanced graduate student with an upto-date report on the state of the art". Originally intended as one volume, the treatise now has four volumes and one (Vol.3) has two parts.

Volume 1 deals with the role of nutrition in pre- and postnatal development, and starts with a discussion of nutrition and the metabolic development of mammals. Metabolic status at various stages of development is closely related to the quality and quantity of the nutrients consumed. The author (Hahn) suggests that the old idea that nutrients act directly on most cells must be discarded and replaced by a concept that, mostly, nutrients have immediate and long-term effects by acting on selected endocrine cells, which then release their hormone to act on target cells. It is this combined effect which controls the development of enzyme systems, cells and tissues.

The development of the brain is important in controlling the ability of the organism to respond to its environment and malnutrition may, because of the timescale over which the brain develops, have permanent deleterious effects upon it. The importance of this subject is reflected in several of the following chapters.

Nutrition and pregnancy is largely discussed from the viewpoint of the effects of disturbances in maternal nutrition on the growth and development of the foetus. The effects of nutrient excesses and of interactions of drugs with nutrients are briefly mentioned. This leads logically to a discussion of breast feeding by Jelliffe and Jelliffe who make the point that the matters that need emphasis are the same the world over.

The contribution on the interactions of nutrition and infection includes a discussion of the problem of the septic patient. In the chapter on the relationship of nutrition to dental development and disease the need is emphasized for more studies to explore the mechanisms and consequences of nutrient deficiencies to oral health. The recognition that atherosclerosis may be a paediatric problem is dealt with. Although this is a prudent approach it is emphasized that proof is at present lacking. A brief chapter is devoted to inborn errors - principles and not details. The final chapter discusses the management of chronic diarrhoea in children.

The second volume, concerned with nutrition and growth, draws attention to the diffuse nature of the subject, the value of having different perspectives, and the fact that there are few "absolute" answers to the various problems such as standards for growth and dietary allowances.

The first chapter considers the problem of nutrient needs. In the absence of proper experiment, the tendency is to consider present intake as "ideal requirements". In the next chapter the complex interrelationships between genetic constitution and nutrition are discussed. The influence of non-dietary factors is dealt with, as also is the problem of metabolic anomalies.

Part II is concerned with the ages of man and is introduced by a chapter on maternofoetal nutrition which presents results of the 1969 longitudinal INCAP study. This leads logically to a consideration of the nutrition of the fullterm and premature baby. The need for further work on the problems of the latter is emphasized. Four chapters are then devoted to the young child and discuss the normal and failure-to-thrive child, proteinenergy malnutrition and obesity respectively. The adolescent, rapidly changing organism and the adult, stabilized situation between development and senescence are then discussed. Part III contains nine contributions which deal with aspects of growth monitoring and nutritional assessment and surveillance, and contains much practical information on different methods and useful tables of reference data.

Volume 3A starts with a discussion of nutrient requirements - what they are and the bases of recommendation - leading on to contributions concerned with energy requirements, the suppliers of energy and their interrelationships, the problem of energy exchange and the partition of food energy. The remaining four chapters deal with nutrients with special functions proteins and amino acids, essential fatty acids, cholesterol and dietary fibre.

Volume 3B is introduced with a chapter on vitamins, mainly those of the B-group, as co-enzymes. There are five chapters on individual vitamins and one on ironhaemoglobin. The chapter entitled "Trace Elements" starts with a somewhat scant treatment of calcium, phosphorus and magnesium. There are useful discussions of drug-nutrient interrelationships and the effects of oral contraceptives on nutrient requirements. The final chapter on nutrition of the elderly has very much a North American bias.

Volume 4 is concerned with the metabolic and clinical application of nutrition. 
The first three chapters discuss the relationship of nutrition to disorders of the haematopoietic, nervous and musculoskeletal systems respectively. These chapters are not exhaustive and are not concerned with therapeutic aspects. The effects of essential nutrients on the gastrointestinal tract and the effects of diseases of the tract on the absorption and utilization of nutrients are dealt with, as also are the nutritional abnormalities occurring in liver disease. Here again there is no discussion of the possible role of nutrition in treatment. This is in contrast to the presentation on cardiac failure which is much concerned with therapeutic aspects and contains a useful table of drug-nutrient interactions of importance in congestive heart failure.

Discussion of the relationship of diet and nutrition to cancer is mainly concerned with their possible involvement in the aetiology of oncological disease, with little mention of the effects of the disease on the nutrition of the patient.

The ageing-nutrition-health triad is discussed by Watkins who points out that education is the prime catalyst for inducing change. The chapter on the endocrine system is concerned with diabetes, vitamin D, and parathyroid and thyroid disorders. Discussion of megavitamins and food fads is largely taken up with doubts about Pauling's well-known views. It is, perhaps, unfortunate that in a text of this sort some consideration is not given to the possibility that larger amounts of vitamins than the currently recommended allowances may have beneficial effects which are difficult to measure and therefore of ten dismissed as anecdotal.

There is a useful and well-referenced chapter on the effects of ethanol. Nutritional aspects of infectious diseases

\section{Abdication of reason} John C. Marshall

The Right Brain: A New Understanding of the Unconscious Mind and its Creative Powers. By T. R. Blakeslee. Pp.275. (Papermac/Doubleday: 1980.) Hardback $£ 10, \$ 10.95$; paperback $£ 2.95$.

THIS is another "popular" book on how to unlock the emotional and creative power of the right cerebral hemisphere. The volume recounts some of the evidence, from both normal and brain-injured people, that has led physicians and clinical psychologists to believe that considerable specialization of function characterizes different areas of the human brain. Blakeslee concentrates on inter- rather than intra-hemispheric localization and leaps somewhat wildly from datum to conclusion. For any reviewer who wants to dip into his repertoire of sarcasm, the volume will provide more than adequate stimulus why is there no woman Beethoven, the author asks, and answers that the cerebral are discussed and it is pointed out that nutritional supportive therapy should be employed to prevent or minimize the depletion of body stores during an infection, and to replace lost nutrients as expeditiously as possible during convalescence. Obesity is the most common nutritional disease in Western society. Assessment, risks and treatment of this poorly understood condition are considered. As a consequence of our poor understanding successful treatment is often elusive, and the demand for treatment has led to a multiplication of fad diets, examples of which are given. The final chapter contains a discussion of the interrelationships of nutrition and the kidney. Effects on kidney function and nutritional disturbances in the nephrotic syndrome, hypertension and renal failure are dealt with, as also are the principles of nutritional therapy.

These volumes are a useful addition to the textbooks available on human nutrition. They are not exhaustive, either in respect of subjects covered or the depth to which each subject is discussed, but the material is well presented and well referenced throughout. Moreover, the books have been produced to a high standard and are a pleasure to read. In spite of their inevitably high price, teachers of nutrition at degree level may well consider it worthwhile acquiring one or more of them. They can be said to have achieved their aim and to present in reasonable compass the present state of the art in a dynamic subject.

J.W.T. Dickerson is Professor of Human Nutrition at the University of Surrey and Consultant Adviser on Clinical Nutrition to the South West Thames Regional Health Authority.

hemispheres of women are functionally less differentiated than those of men. It is tempting to regard Blakeslee's offering as beyond satire and plunge immediately into instant sociology. Why do such books exist? What need do they satisfy?

The "right brain revolution" that Blakeslee and so many others have attempted to explain to a lay audience had its genesis in 1962. Two Los Angeles neurosurgeons, Philip Vogel and Joseph Bogen, revived and refined a surgical procedure for the control of pharmacologically intractable epilepsy. The operation involved sectioning the corpus callosum and the anterior and hippocampal commissures - the main fibre tracts that interconnect the two cerebral hemispheres at the cortical level. There can be little doubt that both the original and subsequent series of commissurotomy operations have been a success; patients whose intellectual and social activity had been severely compromised by generalized convulsions have been able to return to an essentially normal life, although still subject, of course, to the disabilities consequent upon their pre-operative brain damage.

Here we also had a neurosurgical preparation in which, with appropriate techniques that were lacking in the $1940 \mathrm{~s}$, one could investigate the cognitive capacities of the left and right hemispheres isolated from each other except through their brain stem connections. The preparation enabled workers to confirm, extend and fine the deductions that had previously been drawn from the effects of lateralized injury, namely, that the left and right hemispheres (in the majority of the population) are indeed primarily implicated in the exercise of linguistic and visuo-spatial skills, respectively. But because in the commissurotomy patient the left hemisphere has little knowledge of what the right hemisphere is doing (and vice-versa), the enticement of talking about these people as if they had two minds within a single body (brain? person? soul?) proved irresistible (and not only to popularizers). The lure of thinking about the patients in this way is, of course, much more exciting than patiently trying to comprehend the what, how and why of functional localization.

But worse was to come. The disconnected right hemisphere, conspicuously deficient in syntax and verbal reasoning, can yet support dreams, emotions, and a high level of non-verbal memory and intelligence. All that remained was to deify the distinction between the cerebral hemispheres: the left brain became the seat of a cold, calculating, logical and talkative mind, whilst the right brain sustained a warm, intuitive, emotional and artistic mind. In an era in which attitudes towards science are often ambivalent, what could be more attractive than to have science and surgery discover the reality, nay the material substrate even, of intuition, creativity and pure feeling.

Modern times are truly an ideal medium in which to culture the hundreds of articles and books that have attempted to disseminate "the right brain revolution" that Blakeslee describes. Yet there is a sense in which any sociological analysis provides too easy a let-out. Blaming the Zeitgeist never takes us very far. The real vacuum that such books attempt to fill arises from the failure of neuroscientists themselves to provide any significant theoretical account of functional localization. The evidence from anatomy and pathology, and from recordings of the electrical and chemical activity of the normal intact brain - now overwhelmingly supports the notion that distinct cerebral regions are differentially involved in the exercise of higher cognitive functions. How and why this is the case we do not know. It could, however, be an interesting problem for someone to work on.

John C. Marshall is in the Neuropsychology Unit, Department of Clinical Neurology, The Radcliffe Infirmary, Oxford. 